\title{
Anterior scleral thickness changes with accommodation in myopes and emmetropes
}

\author{
Emily C. Woodman-Pieterse ${ }^{a} \quad$ Email: e.pieterse@qut.edu.au \\ Scott A. Read ${ }^{\text {a }}$ \\ Email: sa.read@qut.edu.au \\ Michael J. Collins ${ }^{a}$ \\ Email: m.collins@qut.edu.au \\ David Alonso-Caneiro a \\ Email: d.alonsocaneiro@qut.edu.au \\ ${ }^{a}$ Contact Lens and Visual Optics Laboratory, School of Optometry and Vision Science, \\ Queensland University of Technology, Brisbane, Queensland, Australia
}

Corresponding Author:

Emily Woodman-Pieterse

Contact Lens and Visual Optics Laboratory

School of Optometry and Vision Science

Queensland University of Technology

Room B556, O Block, Victoria Park Road, Kelvin Grove 4059

Brisbane, Queensland, Australia

Word Count: 5776

Number of Tables: 1

Number of Figures: 5

Date of Submission: $7^{\text {th }}$ June 2018 
1 Abstract

2 Although a range of changes in anterior segment structures have been documented

3 to occur during accommodation, the quantification of changes in the structure of the

4 anterior sclera during the accommodation process in human subjects has yet to be

5 examined. This study therefore aimed to investigate the presence of short-term

6 changes in anterior scleral thickness associated with accommodation in young adult

7 myopic and emmetropic subjects. Anterior scleral thickness was measured in 20

8 myopes and 20 emmetropes (mean age $21 \pm 2$ years) during various

9 accommodation demands (0,3 and $6 \mathrm{D})$ with anterior segment optical coherence

10 tomography (AS-OCT). A Badal optometer was mounted in front of the objective

11 lens of the AS-OCT to allow measurements of the anterior temporal sclera $(1,2$ and

$123 \mathrm{~mm}$ posterior to the scleral spur) to be obtained while fixating on an external

accommodation stimulus. Anterior scleral thickness was not statistically different

between refractive groups at baseline, but thinned significantly with the $6 \mathrm{D}$ accommodation demand $(-8 \pm 21 \mu \mathrm{m}, \mathrm{p}<0.05)$, and approached a statistically significant change with the $3 \mathrm{D}$ demand $(-6 \pm 20 \mu \mathrm{m}, \mathrm{p}=0.066)$. While both refractive groups thinned by a statistically significant amount at the $1 \mathrm{~mm}$ location with the 3 D demand; significant $(p<0.001)$ refractive group differences occurred at $3 \mathrm{~mm}$, where the thinning found in the myopic group reached statistical significance with both the $3 \mathrm{D}(-12 \pm 21 \mu \mathrm{m})$ and $6 \mathrm{D}(-19 \pm 17 \mu \mathrm{m})$ demands, and the emmetropes showed no significant changes. This demonstrates the first evidence of a small but statistically significant thinning of the anterior sclera during accommodation. These changes were more prominent in myopes, particularly $3 \mathrm{~mm}$ posterior to the scleral spur. These regional differences may be associated with previously reported regional variations in ciliary body thickness between refractive 
26 groups, regional differences in the contraction of the ciliary muscle with

27 accommodation, or differences in the response of the sclera to these biomechanical

28 forces.

29 Keywords

30 Sclera, accommodation, myopia, optical coherence tomography. 


\section{Introduction}

32 The tough, fibrous sclera is one of the principal determinants of the size and shape

33 of the eye. The biomechanical properties of the normal sclera also allow the eye to resist deformation associated with external forces such as extraocular muscle contraction, and internal forces such as intraocular pressure and accommodation.

There is evidence however, that the normal biomechanical properties of the sclera may be compromised by the development of myopia through a generalised and localised thinning of the sclera (particularly at the posterior pole) (Avetisov et al., 1983; Elsheikh et al., 2010; Norman et al., 2010; Vurgese et al., 2012), associated with elongation of the globe (Rada et al., 2006) which is thought to render the myopic and Essinger, 1994; Troilo and Wallman, 1991; Wallman and Adams, 1987).

In the anterior eye, the sclera is located adjacent to the ciliary body, and these two from the ora serrata where their tendons connect with the equatorial sclera and choroid, and extend anteriorly to their attachment at the scleral spur (Ishikawa, 1962). As the ciliary muscle contracts during accommodation, it draws the mass of the ciliary body inward and forward, allowing the lens to become thicker and more optically powerful (Ishikawa, 1962). Given the sclera's close relationship with the ciliary body, it is conceivable that the forces of contraction during accommodation could influence the structure of the overlying anterior sclera.

53 The apparent link between near work and myopia (see Huang et al., 2015 and

54 Morgan et al., 2018 for comprehensive reviews), has been the catalyst for numerous 
studies that have examined the changes in a variety of ocular parameters during accommodation (Drexler et al., 1998; Mallen et al., 2006; Ostrin et al., 2006; Read et al., 2010; Woodman et al., 2012; Woodman et al., 2011; Zhong et al., 2014), however only qualitative assessment of scleral changes during accommodation have been reported previously (Croft et al., 2013). Since the relatively recent introduction of anterior segment optical coherence tomography (AS-OCT), it has been used to investigate regional variations in anterior scleral thickness (Buckhurst et al., 2015; Ebneter et al., 2015; Pekel et al., 2015; Read et al., 2016b), and variation in anterior scleral thickness with time of day (Read et al., 2016a), contact lens wear (AlonsoCaneiro et al., 2016b), ocular disease (Schlatter et al., 2015; Shoughy et al., 2015; Yoo et al., 2011), and ocular therapeutic procedures (Taban et al., 2010; Zinkernagel et al., 2015). Earlier studies relating to the in vivo determination of human scleral thickness employed ultrasound biomicroscopy (UBM) and magnetic resonance imaging (MRI), which are limited by the requirement for contact with the ocular surface with UBM measures (Oliveira et al., 2006), and lower axial resolution and propensity for motion artefact with MRI measurements (Norman et al., 2010). ASOCT allows for rapid, non-contact imaging of the anterior segment at the higher resolution necessary to detect short-term variations in anterior scleral thickness, and provides excellent repeatability and reliability in the measurement of anterior scleral thickness (Buckhurst et al., 2015; Read et al., 2016a; Read et al., 2016b).

This study aimed to characterise the changes occurring in anterior scleral thickness associated with accommodation using AS-OCT. Given the documented changes in scleral properties with refractive error (Avetisov et al., 1983; Elsheikh et al., 2010; Norman et al., 2010; Rada et al., 2006; Vurgese et al., 2012), both myopic and emmetropic subjects were examined. 


\section{Material and Methods}

\subsection{Participants}

82 Forty healthy young adult participants aged 18-25 years (mean $21 \pm 2$ years) were recruited from the students of the Queensland University of Technology School of

Optometry and Vision Science. Ethics approval was sought from, and granted by the University Human Research Ethics Committee prior to subject recruitment. All participants provided written informed consent before study commencement, and were treated in accordance with the tenets of the Declaration of Helsinki.

Prior to study enrolment, each subject's refractive error, visual acuity, and binocular vision characteristics were determined, to ensure all study participants had a best corrected visual acuity of at least logMAR 0.00 , and amplitudes of accommodation greater than $6 \mathrm{D}$. An anterior and posterior ocular health screening was also conducted for all subjects, to identify and exclude any participant with a history of significant systemic or ocular disease, injury or surgery. A significant portion of the study population wore soft contact lenses regularly (40\%), and were required to cease lens wear at least 24 hours prior to data collection. Any potential participants who wore rigid contact lenses were excluded from the study.

Subjects were categorised into one of two refractive error groups (emmetropic or myopic) by their spherical equivalent refraction (SER), which was obtained via noncycloplegic subjective refraction. Those with SER between -0.25 and $+0.75 \mathrm{D}$ (and $\leq 1 \mathrm{DC})$ were considered emmetropic $(n=20$, mean SER $+0.38 \pm 0.22 \mathrm{D})$, and those with a SER between -0.75 and $-6.00 \mathrm{D}$ (and $\leq 1 \mathrm{DC})$ were classified as myopic $(n=$ 20 , mean SER $-2.83 \pm 1.50 \mathrm{D})$. The refractive groups were well matched in terms of age (emmetropes $21 \pm 1$ years, myopes $22 \pm 2$ years), gender ratio (each group 
comprised of $50 \%$ female, $50 \%$ male), and monocular amplitude of accommodation (emmetropes $11 \pm 1 \mathrm{D}$, myopes $11 \pm 2 \mathrm{D}$ ). Of the 40 subjects recruited for the study, 21 were of Caucasian ethnicity (53\%), 15 were of East/South East Asian ethnicity (37\%), and the remaining 4 subjects (10\%) were from other ethnic origins. The ethnicity of the refractive error groups was also approximately matched (myopes 50\% Caucasian, emmetropes 55\% Caucasian).

\subsection{Instrumentation}

Following the screening and classification of participants, each subject had measures of their left eye's anterior temporal scleral thickness taken during 0,3 and $6 \mathrm{D}$ of accommodation with the Spectralis spectral domain AS-OCT (Heidelberg Engineering, Heidelberg, Germany) utilising the instrument's enhanced depth imaging mode (EDI) to optimise the visualisation of the sclera (Spaide et al., 2008).

The Spectralis AS-OCT uses a super luminescent diode (870nm central wavelength) to capture fast $(40,000 \mathrm{~A}$-scans per second), high resolution images $(3.9 \mu \mathrm{m}$ axial resolution, $11 \mu \mathrm{m}$ lateral resolution) to a scanning depth of $1.9 \mathrm{~mm}$. A scanning laser ophthalmoscope (SLO) is also employed by the instrument to provide en-face images tracked in real-time to enable reliable averaging of the OCT B-scans, improving scan contrast and fine detail.

A Badal optometer was custom built and mounted on the OCT in front of the anterior segment objective lens to allow measurements to be collected while an external fixation target (an LCD screen from an iPhone 4S, Apple Inc., California, USA, screen resolution $326 \mathrm{ppi}$, screen luminance approximately $20 \mathrm{~cd} / \mathrm{m}^{2}$ ) provided varying accommodative demands (Figure 1). The Badal optometer was mounted at a $42^{\circ}$ angle to the measurement axis so that the temporal sclera could be imaged 
while the subject viewed a target on the external LCD screen. The use of a Badal optometer allowed for the correction of each individual's spherical equivalent refractive error, in addition to providing 0,3 and $6 \mathrm{D}$ of accommodative demand. Each subject's right eye was occluded for the duration of the experiment to eliminate the potential confounding effects of convergence in the eye being measured.

For the duration of the experiment, subjects were required to turn their head $42^{\circ}$ to their right to view the LCD screen with their left eye through the Badal optometer system. This head angle allowed for the subject's visual axis to be aligned with the centre of the Badal system, while the OCT was aligned to image the temporal anterior sclera of the left eye. Since no eye movements were required to view the target (only head movement), the potential confounding influence of extraocular muscle forces upon the anterior sclera was reduced. The angle of head turn was set and monitored throughout the task with a smartphone application (AngleFinder, version 1.1, developer Pool Night Studios, LLC) which was used on another iPhone device mounted on a headband and worn on the subject's head. The AngleFinder application utilises the iPhone's in-built gyroscope to measure angles, displaying the roll, pitch and yaw of the device relative to the previous orientation. The yaw was monitored to make sure this did not vary substantially between measurement sessions, thus ensuring consistency in head turn (mean difference in yaw between 0 and 3 , and 0 and $6 \mathrm{D}$ measurements for all subjects was found to be $+1.5 \pm 3^{\circ}$ and $+2 \pm 3^{\circ}$ respectively)

Measures of axial ocular dimensions were also obtained with the Lenstar LS 900 biometer (Haag-Streit AG, Koeniz, Switzerland) during 0, 3 and 6 D of accommodation (using a custom built Badal system and cold mirror to allow fixation of the same Maltese cross target used when imaging the sclera with the AS-OCT) to 
153 investigate any relationship between changes in the sclera and changes in other

154 ocular dimensions.

$155 \quad 2.3$ Methods

156 To eliminate the potential effects of prior near work, subjects were required to watch

157 a movie playing on the LCD screen for 10 minutes, which was imaged through the

158 Badal system at optical infinity. Following this wash-out period, the movie was paused and the screen's display was changed to present a Maltese cross fixation target. The subject was required to fixate the centre of the Maltese cross with their left eye and keep it in sharp focus for the duration of the measurement.

Images of the left anterior temporal sclera were obtained (using the scleral/EDI mode of the OCT), with two $20^{\circ} \times 5^{\circ}(11.1 \mathrm{~mm} \times 2.8 \mathrm{~mm})$ volume scans consisting of 21 lines of 30 averaged B-scans (Figure 2A) taken for each accommodation level, using the instrument's high resolution scanning protocol (1536 x 496 pixels per B-scan). Any scans with a quality index (QI) score $\leq 25 \mathrm{~dB}$ were discarded and retaken.

167 Since the instrument's automatic follow-up function (to register repeated measurements to the same location) is not available during anterior segment imaging, care was taken to position the volume scan in approximately the same vertical and horizontal location on the anterior eye for all repeated image acquisitions. Scleral thickness measures were then derived from the OCT images, defined as the axial distance between the anterior scleral and the posterior scleral boundary (Figure 2B and $2 \mathrm{C}$ ). Although an axial thickness measure is commonly used in OCT analyses, significant B-scan tilts could potentially bias these thickness measures (Alonso-Caneiro et al., 2016a). However analysis of each B-scan revealed minimal tilt between conditions in the images collected (mean tilt of $0.7^{\circ} \pm$ 
$\left.1770.8^{\circ}\right)$, which would result in less than a micron of measurement error in determining 178 scleral thickness in the majority of scans, which is well below the axial resolution of

179 the instrument (3.9 microns). The images were scaled using the instrument's 180 proprietary algorithm which assumed a homogenous refractive index of 1.40 for the 181 sclera to convert optical distances into geometrical measures of tissue thickness 182 (Read et al., 2016b).

After the baseline images were obtained, the Maltese cross target was minimised, and the LCD screen was repositioned to provide a $3 \mathrm{D}$ accommodation demand. The subjects then resumed watching the movie on the screen for a further 10 minutes before it was again paused, and the screen was changed to display the Maltese cross fixation target, and two more OCT measurements were taken with a 3 $\mathrm{D}$ demand. The subjects then underwent another wash-out period, resuming the movie viewing for another 10 minutes with the screen imaged once again at optical infinity. And for the final task, the screen was repositioned to provide a $6 \mathrm{D}$ accommodation stimulus, and the subjects continued watching the movie for 10 minutes, before the OCT measurements were taken again. This protocol was repeated with the Badal optometer mounted on the Lenstar device, allowing 5 measures of ocular biometry of the subjects' left eyes at each of the 3 accommodation levels (0, 3 and $6 \mathrm{D})$.

To reduce the potential confounding influence of diurnal variation in ocular parameters, the measurement sessions were restricted to a 4 hour window each day between 0800-1200 hours (Brown et al., 2009; Chakraborty et al., 2011; Read et al., 2016a; Read et al., 2008). Randomisation of the order of instrument measurements (OCT and Lenstar biometer) was performed prior to the subjects' recruitment to 
ensure any order effects did not confound the results, and all data for an individual subject was collected within the same measurement session on a single day.

\subsection{Data Analysis}

The axial biometry data at each measurement session were analysed using a previously described method (Read et al., 2010; Woodman et al., 2012), and then averaged. The OCT images were exported and analysed using custom written software. Analysis of the en-face SLO images was performed to ensure that the thickness data at each measurement session were registered to the same anterior eye locations. Three common points (e.g. anatomical landmarks such as vessel crossings/bifurcations) were chosen in the en-face image from each volume scan, and the horizontal and vertical translation and rotation required to register each subsequent scan with the baseline scan was derived (Figure 3A). The measurement with better image quality at baseline $(0 \mathrm{D})$ was chosen, and the image at each accommodation level showing the least rotation with respect to this image was chosen for further analysis. Any scans with excessive rotation $\left(>2.9^{\circ}\right.$, which corresponds to rotation that would extend beyond two adjacent scan lines) were excluded to reduce the potential confounding influence of large amounts of data interpolation upon the thickness measurements. One volume scan with acceptable alignment at each accommodation level $(0,3$ and $6 \mathrm{D})$ was required for the subjects to be included in the analysis. The average absolute rotation required for the registration of the scans was $0.2 \pm 1^{\circ}$ for all subjects and all conditions.

Once the rotational and translational alignment between the measurements was determined, the scan corresponding to the position of the central scan line of the baseline scan was identified in the $3 \mathrm{D}$ and $6 \mathrm{D}$ scans (Figure 3B). The central 
scans and the two consecutive scans either side of this (i.e. the 5 central scans) in each measurement were then analysed to segment the posterior scleral boundary, the anterior scleral boundary and anterior conjunctival boundary in each scan (Figure 2C and $3 \mathrm{C}$ ). Each scan was initially segmented using an automated algorithm which delineated the anterior conjunctival surface and the posterior scleral surface.

One experienced observer, masked to the subjects' refractive error and accommodation level then checked the integrity of the automated segmentation, and manually corrected any errors. The masked observer then manually segmented the boundary between the anterior sclera and episclera, which was identified based upon the position of the episcleral blood vessels in the scan. In accordance with previous studies using AS-OCT to examine anterior scleral thickness, the deep episcleral vascular plexus was identified as a thin, hyporeflective region external to the solid scleral tissue (Alonso-Caneiro et al., 2016b; Ebneter et al., 2015; Read et al., 2016a; Read et al., 2016b; Shoughy et al., 2015). The position of the scleral spur in each OCT image was also manually marked, according to previously defined criteria (Day et al., 2013; Sakata et al., 2008).

241 The data obtained via the segmentation of the five central scan lines in each measurement were then used to derive a thickness volume map of both the temporal total anterior wall thickness (from the anterior conjunctiva to the posterior sclera) and the scleral thickness (from the anterior sclera to the posterior sclera) for each subject at each accommodation level (Figure 3C).

246 Each thickness volume (for $3 \mathrm{D}$ and $6 \mathrm{D}$ ) was then rotated using custom written software in order to precisely align/register each thickness volume to the baseline ( 0 D) measurement using the rotational information derived from the analysis of the en249 face images (Figure 3D). The thickness profile from the central line, in each of the 
250 final rotated thickness volumes was then extracted and analysed, thus ensuring that

251 the thickness profile from each condition was derived from the same anterior eye

252 location. The total anterior wall thickness and scleral thickness at discrete points 1 ,

2532 and $3 \mathrm{~mm}$ posterior to the scleral spur were calculated, measured in the

254 axial/vertical direction of the OCT images from the anterior conjunctival boundary to

255 the anterior scleral boundary, and the anterior scleral boundary to the posterior

256 scleral boundary, respectively. These locations were chosen for comparability to

257 previous OCT studies examining regional scleral thickness (Buckhurst et al., 2015;

258 Pekel et al., 2015; Read et al., 2016a; Read et al., 2016b), and changes in the ciliary

259 body associated with accommodation and refractive error (Buckhurst et al., 2013;

260 Kuchem et al., 2013; Lewis et al., 2012; Lossing et al., 2012; Pucker et al., 2013;

261 Richdale et al., 2013) that also used fixed distances from the scleral spur.

262 Reliability and repeatability of the segmentation of the sclera, episclera and

263 conjunctiva was assessed by having the experienced observer analyse five

264 randomly selected scans three times each. The average within session standard

265 deviation for the determination of the location of the scleral spur was $2 \mu \mathrm{m}$. For the

266 total wall thickness the within session SD across all measurement locations ranged

267 between $3 \mu \mathrm{m}$ and $5 \mu \mathrm{m}$; and for the scleral thickness ranged between $4 \mu \mathrm{m}$ and 7

$268 \mu \mathrm{m}$. The intraclass correlation coefficient (two-way mixed model, absolute

269 agreement) for intra-observer reliability, was greater than 0.96 for all parameters

270 tested (Shrout and Fleiss, 1979).

271 Of the 40 subjects examined, seven were excluded from analysis of the OCT images

272 (2 myopes, 5 emmetropes). Five of the subjects were excluded due to large

273 amounts of rotation/cyclotorsion between measurement conditions, meaning that

274 identical regions of the sclera were not imaged. The quality of all of the OCT B-scan 
275 images were also checked, and one subject was excluded due to prominent

276 shadows on the OCT images from their lashes which obscured much of the detail in

277 their images, and one subject was excluded because they were unable to keep their

278 fixation steady enough to have all their measurements taken. Of the remaining 33

279 subjects, the average QI of the scans was $40 \pm 3 \mathrm{~dB}$ out of a possible $50 \mathrm{~dB}$.

\subsection{Statistical Analysis}

281 A repeated measures ANOVA was performed to examine the influence of accommodation level (0, 3, $6 \mathrm{D})$ and measurement location $(1 \mathrm{~mm}, 2 \mathrm{~mm}, 3 \mathrm{~mm}$ from the scleral spur) on both scleral thickness and total wall thickness (within subject factors), and for any differences associated with refractive error group (between subject factor). If significant differences were identified $(p<0.05)$, posthoc testing with Bonferroni correction was performed. An analysis of covariance (ANCOVA) was also performed to identify any associations between the changes in total wall or scleral thickness and the changes in other measured biometric parameters (e.g. axial length) during accommodation using the methods of Bland and Altman (Bland and Altman, 1995) for the analysis of repeated measures.

\section{Results}

The baseline scleral and total anterior wall thicknesses were not significantly different between the refractive groups, but did show significant variation with distance from the scleral spur $(p<0.001)$ (Table 1). Anterior scleral thickness was significantly greater at the $3 \mathrm{~mm}$ location $(543 \pm 67 \mu \mathrm{m})$ compared to the $1 \mathrm{~mm}(512$ $\pm 52 \mu \mathrm{m})(\mathrm{p}<0.05)$ and $2 \mathrm{~mm}$ locations $(504 \pm 60 \mu \mathrm{m})(p<0.001)$. Likewise, the 
total anterior wall thickness varied significantly with measurement location $(p<0.05)$, although in this case it was the $2 \mathrm{~mm}$ region $(730 \pm 87 \mu \mathrm{m})$ that was significantly thinner than the $1 \mathrm{~mm}(751 \pm 81 \mu \mathrm{m})(\mathrm{p}<0.05)$ and $3 \mathrm{~mm}(752 \pm 91 \mu \mathrm{m})(\mathrm{p}<0.001)$ regions.

Anterior scleral thickness changed significantly with accommodation, with pairwise comparisons revealing significant scleral thinning with accommodation to the $6 \mathrm{D}$ stimulus (mean change $-8 \pm 21 \mu \mathrm{m}, \mathrm{p}<0.05$ ), and thinning which approached significance during accommodation to the $3 \mathrm{D}$ stimulus $(-6 \pm 20 \mu \mathrm{m}, \mathrm{p}=0.066)$ (Figure 4).

The amount of scleral thickness change with accommodation varied significantly with refractive group, with significant thickness changes in the myopic sclerae recorded at both the 3 and $6 \mathrm{D}$ accommodation demands $(p<0.05)$, but no significant change in the emmetropic group at either accommodation level. A significant difference between the refractive groups' mean scleral thickness change was observed at the 3 D level $(p<0.05)$, with a scleral thinning seen in the myopes $(-12 \pm 18 \mu \mathrm{m})$, while the emmetropic mean scleral thickness remained unchanged $(+1 \pm 20 \mu \mathrm{m}, \mathrm{p}<0.05)$. However, during accommodation to the $6 \mathrm{D}$ stimulus statistically significant refractive group differences were not evident $(p=0.199)$, with both the myopes $(-11 \pm 24 \mu \mathrm{m})$ and emmetropes $(-5 \pm 17 \mu \mathrm{m})$ thinning on average by similar magnitudes.

Change in anterior scleral thickness during accommodation also varied significantly $(p<0.05)$ with measurement location $(1,2,3 \mathrm{~mm}$ posterior to the scleral spur $)$. When all subjects were considered, a highly significant thinning was observed at the $1 \mathrm{~mm}$ location during accommodation to the $3 \mathrm{D}$ demand $(-13 \pm 16 \mu \mathrm{m}, \mathrm{p}<0.001)$, 
and a significant thinning observed at the $3 \mathrm{~mm}$ location with the $6 \mathrm{D}$ demand $(-11 \pm$ $18 \mu \mathrm{m}, \mathrm{p}<0.05)$ (Figure 5A and 5B).

The change in mean scleral thickness with accommodation by region by refractive group interaction approached significance $(p=0.055)$. Both the myopic $(-15 \pm 17$ $\mu \mathrm{m})$ and emmetropic $(-10 \pm 15 \mu \mathrm{m})$ participants thinned in the $1 \mathrm{~mm}$ region during accommodation to the $3 \mathrm{D}$ stimulus, and the myopes additionally thinned in the 3 $\mathrm{mm}$ region during accommodation to both the $3 \mathrm{D}(-12 \pm 21 \mu \mathrm{m})$ and $6 \mathrm{D}(-19 \pm 17$ $\mu \mathrm{m})$ stimuli. Significant mean scleral thickening was observed in the emmetropic participants in the $3 \mathrm{~mm}$ region with the $3 \mathrm{D}(+11 \pm 15 \mu \mathrm{m}, \mathrm{p}<0.05)$, but not $6 \mathrm{D}(-1$ $\pm 3 \mu \mathrm{m}, \mathrm{p}>0.05)$ accommodation demands. These refractive group differences in the change in mean scleral thickness were significant $(p=0.001)$ in the $3 \mathrm{~mm}$ region with accommodation to both the 3 and $6 \mathrm{D}$ stimuli (Figure $5 \mathrm{~A}$ and $5 \mathrm{~B}$ ).

On average, the total anterior wall thickness thinned significantly for all subjects with the $6 \mathrm{D}$ (mean change $-6 \pm 14 \mu \mathrm{m}, p<0.05$ ), but not the $3 \mathrm{D}$ (mean change $-4 \pm 14$ $\mu \mathrm{m}, \mathrm{p}=0.088$ ) accommodation demand (Figure 4). A significant accommodation by region interaction was observed, with both the $2 \mathrm{~mm}(-6 \pm 12 \mu \mathrm{m}, \mathrm{p}<0.05)$ and 3 $\mathrm{mm}(-7 \pm 14 \mu \mathrm{m}, \mathrm{p}<0.05)$ locations thinning significantly from baseline with the $6 \mathrm{D}$ accommodation demand. Both refractive groups showed a similar amount of total anterior wall thinning with accommodation $(p=0.471)$ (Figure 5C and 5D).

All subjects exhibited significant $(p<0.001)$ anterior chamber depth $(A C D)$ shallowing (mean change of $-0.10 \pm 0.05 \mathrm{~mm}$ and $-0.29 \pm 0.06 \mathrm{~mm}$, for the $3 \mathrm{D}$ and $6 \mathrm{D}$ demands respectively) and increased lens thickness (LT) with accommodation (mean change of $+0.11 \pm 0.05 \mathrm{~mm}$ and $+0.30 \pm 0.06 \mathrm{~mm}$ ), confirming the accommodative response for the $3 \mathrm{D}$ and $6 \mathrm{D}$ demands. ANCOVA revealed that the 
scleral thinning seen at the $3 \mathrm{~mm}$ location during accommodation was significantly positively correlated with the ACD shallowing $\left(p<0.05, r^{2}=0.138\right.$, slope $\left.\beta=3.74\right)$ and significantly negatively correlated with the lens thickening $\left(p<0.05, r^{2}=0.13\right.$, slope $\beta=-3.797$ ). The scleral thinning at the $3 \mathrm{~mm}$ location was also weakly negatively correlated with the change in $\mathrm{AL}$ with accommodation $\left(p=0.058, r^{2}=\right.$ 0.054 , slope $\beta=-0.18$ ), although this association only approached significance.

\section{Discussion}

This study provides the first quantitative evidence that the anterior sclera undergoes a small but statistically significant thinning with accommodation in young adults. The similarities between the thickness changes seen in the sclera and total anterior wall with 3 and $6 \mathrm{D}$ of accommodation (Figure 4) suggest that the changes observed with accommodation appear to be primarily confined to the sclera, with minimal change seen in the overlying conjunctiva and episclera in the majority of measurement locations. These changes in anterior scleral thickness appeared more prominent in the myopic than emmetropic subjects. While the myopes exhibited statistically significant thinning at both 3 and $6 \mathrm{D}$ accommodation demands, the emmetropes demonstrated less magnitude of change, and their overall scleral thickness (averaged across the 3 measurement locations) was not significantly different from baseline with any level of accommodation. The scleral changes with accommodation also varied dependent upon the region of measurement, with a statistically significant thinning seen $1 \mathrm{~mm}$ posterior to the scleral spur in both the myopes and emmetropes, and the myopes also showing statistically significant thinning $3 \mathrm{~mm}$ posterior to the scleral spur with increased accommodation levels. 
369 Previous studies utilising time-domain AS-OCT have reported that the ciliary muscle 370 of both children (Lewis et al., 2012) and adults (Lossing et al., 2012; Sheppard and

371 Davies, 2010) undergoes an overall shortening during accommodation, with the 372 anterior portion of the muscle thickening and the ciliary body moving forwards and 373 inwards. While the ciliary muscle thickens in the anterior region $1 \mathrm{~mm}$ posterior to 374 the scleral spur, the posterior ciliary muscle is reported to thin, particularly in the 375 location $3 \mathrm{~mm}$ posterior to the scleral spur (Lewis et al., 2012; Lossing et al., 2012). 376 Since the longitudinal ciliary muscle fibres are firmly attached to the overlying sclera 377 at both the scleral spur and ora serata (Ishikawa, 1962), it is possible that the mechanical force from the ciliary muscle could directly or indirectly influence the scleral structure and lead to the scleral thickness changes that we observed during accommodation.

Given that the ciliary body transfers its mass anteriorly during contraction, it is possible that the thinning observed in the most anterior portion of the sclera that we examined (1 $\mathrm{mm}$ location) during accommodation to the $3 \mathrm{D}$ stimulus, is occurring in response to the ciliary body movement adjacent to this region of the sclera. However, the change in scleral thickness at the $1 \mathrm{~mm}$ location did not increase in magnitude with the larger accommodation demand, which would be expected if direct mechanical force from the ciliary muscle acting on the sclera was the only mechanism involved.

Another possible mechanism by which the anterior sclera may thin during accommodation is through the action of scleral myofibroblast cells. Myofibroblasts have been identified in the sclera of humans and monkeys and are thought to contract in response to external scleral tissue stresses (e.g. from eye movements, accommodation and fluctuations in intraocular pressure) to prevent deformation of 
394 the surrounding extracellular matrix (Poukens et al., 1998). These cells are found within the inner sclera of the posterior pole (Poukens et al., 1998), and in the region of the scleral spur of human eyes (Tamm et al., 1995). The scleral thinning seen at the $1 \mathrm{~mm}$ location with accommodation in both the myopes and emmetropes may

therefore relate to a contractile response from the myofibroblasts within the scleral spur. As the ciliary body contracts during accommodation, it presumably imposes mechanical force upon on the anterior sclera overlying the ciliary body region. The myofibroblasts within the scleral spur may then contract in response to these forces and result in a slight thinning of the sclera within this region.

There are structural differences in the ciliary body of myopic and emmetropic eyes, with numerous studies reporting thicker posterior ciliary muscles in the eyes of myopes compared to emmetropes or hyperopes (Bailey et al., 2008; Kuchem et al., 2013; Lewis et al., 2012; Muftuoglu et al., 2009; Oliveira et al., 2005; Pucker et al., 2013). Most studies report the greatest difference in thickness associated with myopia occurs in the region $3 \mathrm{~mm}$ posterior to the scleral spur (Lewis et al., 2012), although some studies also report similar thickening at the $2 \mathrm{~mm}$ location (Bailey et al., 2008; Buckhurst et al., 2013; Muftuoglu et al., 2009; Oliveira et al., 2005; Pucker et al., 2013), with longer eyes demonstrating thicker ciliary muscles. These findings show some parallels with the data presented in our study. The greatest changes in scleral thickness with accommodation were observed in the myopic subjects and in the region of the sclera located $3 \mathrm{~mm}$ posterior to the scleral spur, which corresponds to the region of the ciliary body that is reportedly thickest in myopes (Bailey et al., 2008; Buckhurst et al., 2013; Lewis et al., 2012; Muftuoglu et al., 2009; Oliveira et al., 2005; Pucker et al., 2013). In the emmetropic subjects however, the greatest scleral thinning was found in the region $1 \mathrm{~mm}$ posterior to the scleral spur, 
which corresponds to the area of the ciliary body which is reported to be comparatively thicker in emmetropes and hyperopes (Kuchem et al., 2013; Pucker et al., 2013). It is therefore possible that the scleral thinning we have found accompanying accommodation is due to contraction of the ciliary muscle adjacent to the sclera, with greater thinning seen in the regions of the sclera which correspond to the thickest regions of the ciliary body for that particular refractive group.

The disparity in the scleral thinning response with accommodation between the refractive groups that we found may reflect the reported change in scleral biomechanical properties that accompany myopia. Investigations regarding the biomechanical changes of the sclera in response to myopia development in animals have found an increase in the viscoelasticity of the sclera of form deprived tree shrews, making these myopic sclerae weaker and more extensible, and susceptible to deformation under otherwise normal scleral stressors such as intraocular pressure (Phillips et al., 2000; Siegwart and Norton, 1999). This may explain why scleral thinning was more pronounced in the myopes than the emmetropes at both the $3 \mathrm{D}$ and $6 \mathrm{D}$ accommodation levels, with the sclera of the myopic eyes appearing to be more readily deformed under normal accommodative forces. The regional variation in scleral thinning reported in this study may be due to differences in the biomechanical properties of the anterior sclera as it moves from the limbal to equatorial region, since evidence of a variation in scleral resistance has previously been reported between the anterior scleral quadrants (Patel et al., 2011). The emmetropic group not only demonstrated less overall scleral thinning with accommodation than the myopic group, but also demonstrated a small but statistically significant scleral thickening at the $3 \mathrm{~mm}$ location in response to the $3 \mathrm{D}$ accommodation demand. While the exact mechanism underlying this small 
magnitude scleral thickening is unclear, we speculate that this is possibly due to a redistribution of anterior scleral tissue during accommodation. There could be a complex interaction between the forces imposed on the sclera by contraction of the ciliary body and myofibroblasts within the scleral spur, combined with regional and refractive group variations in the ciliary body and scleral resistance leading to thickening in a region in one group of subjects, and thinning in another group.

Structurally the myopic sclera is thought to be generally thinner than non-myopic eyes, particularly in posterior ocular regions, with highly myopic eyes exhibiting areas of localised ectasia through pronounced scleral thinning at the posterior pole (Avetisov et al., 1983; Elsheikh et al., 2010; Norman et al., 2010; Vurgese et al., 2012). Despite these reports, the baseline scleral thickness values of the two refractive groups used in this study were not significantly different. It is possible that our myopic subjects did not have sufficient axial elongation to produce significant anterior scleral thinning. Another recent study utilising AS-OCT also reported no difference in anterior temporal scleral thickness between age-matched high myopes and emmetropic controls (Pekel et al., 2015). This suggests that any structural changes in the anterior sclera associated with myopia are minimal, which is consistent with previous studies reporting scleral thinning associated with myopia largely confined to the posterior eye (Avetisov et al., 1983; Elsheikh et al., 2010; Norman et al., 2010; Vurgese et al., 2012).

The correlation found between the changes in the scleral thickness at the $3 \mathrm{~mm}$ location and the anterior chamber and lens thickness during accommodation indicates that the changes seen in the $3 \mathrm{~mm}$ region are related to the magnitude of the accommodation response. The magnitude of anterior chamber shallowing and lens thickening during accommodation observed in our current study are consistent 
with previous reports of the biometric accommodation response in young adults (Ostrin et al., 2006). While this finding suggests that our subjects demonstrated a consistent accommodation response during the scleral measurements (given that the same Badal system and target was used in both parts of the study), a limitation of this study is that simultaneous measures of accommodative response were not collected during the OCT scleral measurements. Asymmetries have previously been been observed between the nasal and temporal ciliary body in both animals (Glasser, Croft, Brumback, \& Kaufman, 2001), and humans in vitro (Aiello, Tran, \& Rao, 1992) and in vivo (Sheppard and Davies, 2011). Sheppard and Davies (Sheppard and Davies, 2011) reported the temporal ciliary muscle is thicker and has a greater ability to thin than the nasal ciliary muscle, and interpreted this to indicate a stronger contractile response in the temporal region. Comparing differences in anterior scleral changes during accommodation between the nasal and temporal regions may strengthen the argument that the force of contraction of the underlying ciliary muscle leads to this transient scleral thinning. While it was not possible to obtain nasal AS-OCT scans with the participants fixating in primary gaze in our study due to the position of the nose, future studies which overcome this limitation would provide additional insights into the mechanism underlying the anterior scleral thickness changes during accommodation.

Despite many of the changes in scleral thickness noted during accommodation reaching statistical significance, it is important to acknowledge that a lot of these significant changes were small in magnitude, and close to the axial resolution of the Spectralis AS-OCT instrument. Future work on this topic with instrumentation with higher resolution capabilities may be necessary to confirm our findings. 


\section{Conclusions}

495 In conclusion, this study demonstrates for the first time a thinning of the anterior

496 temporal sclera during accommodation that is more prominent in young adult

497 myopes. Statistically significant regional differences in the scleral response also

498 accompanied accommodation, with greater thinning seen in the region $3 \mathrm{~mm}$

499 posterior to the scleral spur in myopic subjects, which increased with greater

500 accommodation demand. These regional differences may be explained by

501 previously reported regional differences in the ciliary body thickness between

502 refractive groups, and/or regional differences in the biomechanical properties of the

503 sclera between refractive groups. 
504

0

\section{Acknowledgements}

The authors thank Mr Brett Davis for his assistance with the design and construction of the mounted Badal optometer and cold mirror systems. Some aspects of this study were presented at the International Myopia Conference (IMC) 2015 meeting.

Disclosure: E.C. Woodman-Pieterse, None; S.A. Read, None; M.J. Collins, None; D. Alonso-Caneiro, None.

\section{References}

Aiello, A.L., Tran, V.T., Rao, N.A., 1992. Postnatal development of the ciliary body and pars plana. Archives of Ophthalmology 110, 802-805.

Alonso-Caneiro, D., Read, S.A., Vincent, S.J., Collins, M.J., Mojtkowski, M., 2016a.

Tissue thickness calculation in ocular optical coherence tomography. Biomedical Optics Express 7, 629-645.

Alonso-Caneiro, D., Vincent, S.J., Collins, M.J., 2016b. Morphological changes in the conjunctiva, episclera and sclera following short-term miniscleral contact lens wear in rigid lens neophytes. Contact Lens and Anterior Eye 39, 53-61.

Avetisov, E.S., Savitskaya, N.F., Vinetskaya, M.I., Iomdina, E.N., 1983. A study of biochemical and biomechanical qualities of normal and myopic eye sclera in humans of different age groups. Metabolic, Pediatric, and Systemic Ophthalmology 7, 183188.

Bailey, M.D., Sinnott, L.T., Mutti, D.O., 2008. Ciliary body thickness and refractive error in children. Investigative Ophthalmology and Visual Science 49, 4353-4360. 
526 Bland, J.M., Altman, D.G., 1995. Calculating correlation coefficients with repeated 527 observations: part 1 - correlation within subjects British Medical Journal 310, 446.

528 Brown, J.S., Flitcroft, D.I., Ying, G.S., Francis, E.L., Schmid, G.F., Quinn, G.E.,

529 Stone, R.A., 2009. In vivo human choroidal thickness measurements: evidence for 530 diurnal fluctuations. Investigative Ophthalmology and Visual Science 50, 5-12.

531 Buckhurst, H., Gilmartin, B., Cubbidge, R.P., Nagra, M., Logan, N.S., 2013. Ocular 532 biometric correlates of ciliary muscle thickness in human myopia. Ophthalmic and 533 Physiological Optics 33, 294-304.

534 Buckhurst, H.D., Gilmartin, B., Cubbidge, R.P., Logan, N.S., 2015. Measurement of 535 scleral thickness in humans using anterior segment optical coherent tomography. 536 PLoS One 10, e0132902.

537 Chakraborty, R., Read, S.A., Collins, M.J., 2011. Diurnal variations in axial length, 538 choroidal thickness, intraocular pressure, and ocular biometrics. Investigative 539 Ophthalmology and Visual Science 52, 5121-5129.

540 Croft, M.A., Nork, T.M., McDonald, J.P., Katz, A., Lütjen-Drecoll, E., Kaufman, P.L., 541 2013. Accommodative movements of the vitreous membrane, choroid, and sclera in 542 young and presbyopic human and nonhuman primate eyes. Investigative 543 Ophthalmology and Visual Science 54, 5049-5058.

544 Day, A.C., Garway-Heath, D., Broadway, D.C., Jiang, Y., Hayat, S., Dalzell, N., 545 Khaw, K.T., Foster, P.J., 2013. Spectral domain optical coherence tomography 546 imaging of the aqueous outflow structures in normal participants of the EPIC-Norfolk 547 Eye Study. British Journal of Ophthalmology 97, 189-195.

548 Drexler, W., Findl, O., Schmetterer, L., Hitzenberger, C.K., Fercher, A.F., 1998. Eye 549 elongation during accommodation in humans: differences between emmetropes and 550 myopes. Investigative Ophthalmology and Visual Science 39, 2140-2147. 
551 Ebneter, A., Häner, N.U., Zinkernagel, M.S., 2015. Metrics of the normal anterior

552 sclera: imaging with optical coherence tomography. Graefe's Archive for Clinical and

553 Experimental Ophthalmology 253, 1575-1580.

554 Elsheikh, A., Geraghty, B., Alhasso, D., Knappett, J., Campanelli, M., Rama, P.,

555 2010. Regional variation in the biomechanical properties of the human sclera.

556 Experimental Eye Research 90, 624-633.

557 Glasser, A., Croft, M.A., Brumback, L., Kaufman, P.L., 2001. Ultrasound

558 biomicroscopy of the aging rhesus monkey ciliary region. Optometry and Vision

559 Science 78, 417-424.

560 Huang, H.M., Chang, D.S., Wu, P.C., 2015. The association between near work

561 activities and myopia in children - a systematic review and meta-analysis. PLoS

562 ONE 10, e0140419.

563 Ishikawa, T., 1962. Fine structure of the human ciliary muscle. Investigative

564 Ophthalmology and Visual Science 1, 587-608.

565 Kuchem, M.K., Sinnott, L.T., Kao, C.Y., Bailey, M.D., 2013. Ciliary muscle thickness

566 in anisometropia. Optometry and Vision Science 90, 1312-1320.

567 Lewis, H.A., Kao, C.Y., Sinnott, L.T., Bailey, M.D., 2012. Changes in ciliary muscle

568 thickness during accommodation in children. Optometry and Vision Science 89, 727-

569737.

570 Lossing, L.A., Sinnott, L.T., Kao, C.Y., Richdale, K., Bailey, M.D., 2012. Measuring

571 changes in ciliary muscle thickness with accommodation in young adults. Optometry

572 and Vision Science 89, 719-726.

573 Mallen, E.A., Kashyap, P., Hampson, K.M., 2006. Transient axial length change

574 during the accommodation response in young adults. Investigative Ophthalmology

575 and Visual Science 47, 1251-1254. 
576 Morgan, I.G., French, A.N., Ashby, R.S., Guo, X., Ding, X., He, M., Rose, K.A., 2018.

577 The epidemics of myopia: aetiology and prevention. Progress in Retinal and Eye

578 Research 62, 134-149.

579 Muftuoglu, O., Hosal, B.M., Zilelioglu, G., 2009. Ciliary body thickness in unilateral 580 high myopia. Eye 23, 1176-1181.

581 Norman, R.E., Flanagan, J.G., Rausch, S.M., Sigal, I.A., Tertinegg, I., Eilaghi, A., 582 Portnoy, S., Sled, J.G., Ethier, C.R., 2010. Dimensions of the human sclera:

583 thickness measurement and regional changes with axial length. Experimental Eye 584 Research 90, 277-284.

585 Norton, T.T., Essinger, J.A.M., N.A., 1994. Lid-suture myopia in tree shrews with 586 retinal ganglion cell blockade. Vision Neuroscience 11, 143-153.

587 Oliveira, C., Tello, C., Liebmann, J., Ritch, R., 2006. Central corneal thickness is not 588 related to anterior scleral thickness or axial length. Journal of Glaucoma 15, 190589194.

590 Oliveira, C., Tello, C., Liebmann, J.M., Ritch, R., 2005. Ciliary body thickness 591 increases with increasing axial myopia. American Journal of Ophthalmology 140, $592 \quad 324-325$.

593 Ostrin, L., Kasthurirangan, S., Win-Hall, D., Glasser, A., 2006. Simultaneous 594 measurements of refraction and A-scan biometry during accommodation in humans. 595 Optometry and Vision Science 83, 657-665.

596 Patel, H., Gilmartin, B., Cubbidge, R.P., Logan, N.S., 2011. In vivo measurement of 597 regional variation in anterior scleral resistance to Schiotz indentation. Ophthalmic 598 and Physiological Optics 31, 437-443. 
599 Pekel, G., Yagci, R., Acer, S., Ongun, G.T., Cetin, E.N., Simavli, H., 2015.

600 Comparison of corneal layers and anterior sclera in emmetropic and myopic eyes.

601 Cornea 34, 786-790.

602 Phillips, J.R., Khalaj, M., McBrien, N.A., 2000. Induced myopia associated with

603 increased scleral creep in chick and tree shrew eyes. Investigative Ophthalmology

604 and Visual Science 41, 2028-2034.

605 Poukens, V., Glasgow, B.J., Demer, J.L., 1998. Nonvascular contractile cells in

606 sclera and choroid of humans and monkeys. Investigative Ophthalmology and Visual

607 Science 39, 1765-1774.

608 Pucker, A.D., Sinnott, L.T., Kao, C.Y., Bailey, M.D., 2013. Region-specific

609 relationships between refractive error and ciliary muscle thickness in children.

610 Investigative Ophthalmology and Visual Science 54, 4710-4716.

611 Rada, J.A., Shelton, S., Norton, T.T., 2006. The sclera and myopia. Experimental

612 Eye Research 82, 185-200.

613 Read, S.A., Alonso-Caneiro, D., Free, K.A., Labuc-Spoors, E., Leigh, J.K., Quirk,

614 C.J., Yang, Z.Y.L., Vincent, S.J., 2016a. Diurnal variation of anterior scleral and

615 conjunctival thickness. Ophthalmic and Physiological Optics 36, 279-289.

616 Read, S.A., Alonso-Caneiro, D., Vincent, S.J., Bremner, A., Fothergill, A., Ismail, B.,

617 McGraw, R., Quirk, C.J., Wrigley, E., 2016b. Anterior eye tissue morphology: Scleral

618 and conjunctival thickness in children and young adults. Scientific Reports 6, 33796.

619 Read, S.A., Collins, M.J., Iskander, D.R., 2008. Diurnal variation of axial length,

620 intraocular pressure, and anterior eye biometrics. Investigative Ophthalmology and

621 Visual Science 49, 2911-2918. 
622 Read, S.A., Collins, M.J., Woodman, E.C., Cheong, S.H., 2010. Axial length changes 623 during accommodation in myopes and emmetropes. Optometry and Vision Science $62487,656-662$.

625 Richdale, K., Sinnott, L.T., Bullimore, M.A., Wassenaar, P.A., Schmalbrock, P., Kao, 626 C.Y., Patz, S., Mutti, D.O., Glasser, A., Zadnik, K., 2013. Quantification of age627 related and per dioptre accommodative changes of the lens and ciliary muscle in the 628 emmetropic human eye. Investigative Ophthalmology and Visual Science 54, 10956291105.

630 Sakata, L.M., Lavanya, R., Friedman, D.S., Aung, H.T., Seah, S.K., Foster, P.J., 631 Aung, T., 2008. Assessment of the scleral spur in anterior segment optical 632 coherence tomography images. Archives of Ophthalmology 126, 181-185.

633 Schlatter, B., Beck, M., Frueh, B.E., Tappeiner, C., Zinkernagel, M., 2015. Evaluation 634 of scleral and corneal thickness in keratoconus patients. Journal of Cataract and 635 Refractive Surgery 41, 1073-1080.

636 Sheppard, A.L., Davies, L.N., 2010. In vivo analysis of ciliary muscle morphologic 637 changes with accommodation and axial ametropia. Investigative Ophthalmology and 638 Visual Science 51, 6882-6889.

639 Sheppard, A.L., Davies, L.N., 2011. The effect of ageing on in vivo human ciliary 640 muscle morphology and contractility. Investigative Ophthalmology and Visual 641 Science 52, 1809-1816.

642 Shoughy, S.S., Jaroudi, M.O., Kozak, I., Tabbara, K.F., 2015. Optical coherence 643 tomography in the diagnosis of scleritis and episcleritis. American Journal of 644 Ophthalmology 159, 1045-1049.

645 Shrout, P.E., Fleiss, J.L., 1979. Intraclass correlations: uses in assessing rater 646 reliability. Psychological Bulletin 86, 420-428. 
647 Siegwart, J.T.J., Norton, T.T., 1999. Regulation of the mechanical properties of tree 648 shrew sclera by the visual environment. Vision Research 39, 387-407.

649 Spaide, R.F., Koizumi, H., Pozzoni, M.C., 2008. Enhanced depth imaging spectral650 domain optical coherence tomography. American Journal of Ophthalmology 146, $651 \quad 496-500$.

652 Taban, M., Lowder, C.Y., Ventura, A.A., Sharma, S., Nutter, B., Hayden, B.C., 653 Dupps, W.J., Kaiser, P.K., 2010. Scleral thickness following fluocinolone acetonide 654 implant (Retisert). Ocular Immunology and Inflammation 18, 305-313.

655 Tamm, E.R., Koch, T.A., Mayer, B., Stefani, F.H., Lütjen-Drecoll, E., 1995.

656 Innervation of myofibroblast-like scleral spur cells in human and monkey eyes.

657 Investigative Ophthalmology and Visual Science 36, 1633-1644.

658 Troilo, D., Wallman, J., 1991. The regulation of eye growth and refractive state: an 659 experimental study of emmetropization. Vision Research 31, 1237-1250.

660 Vurgese, S., Panda-Jonas, S., Jonas, J.B., 2012. Scleral thickness in human eyes. 661 PLoS One 7, e29692.

662 Wallman, J., Adams, J.I., 1987. Developmental aspects of experimental myopia in 663 chicks: susceptibility, recovery and relation to emmetropization. Vision Research 27, $664 \quad 1139-1163$.

665 Woodman, E.C., Read, S.A., Collins, M.J., 2012. Axial length and choroidal 666 thickness changes accompanying prolonged accommodation in myopes and 667 emmetropes. Vision Research 72, 34-41.

668 Woodman, E.C., Read, S.A., Collins, M.J., Hegarty, K.J., Priddle, S.B., Smith, J.M., 669 Perro, J.V., 2011. Axial elongation following prolonged near work in myopes and 670 emmetropes. British Journal of Ophthalmology 95, 652-656. 
671 Yoo, C., Eom, Y.S., Suh, Y.W., Kim, Y.Y., 2011. Central corneal thickness and

672 anterior scleral thickness in Korean patients with open-angle glaucoma: an anterior

673 segment optical coherence study. Journal of Glaucoma 20, 95-99.

674 Zhong, J., Tao, A., Xu, Z., Jiang, H., Shao, Y., Zhang, H., Liu, C., Wang, J., 2014.

675 Whole eye axial biometry during accommodation using ultra-long scan depth optical

676 coherence tomography. American Journal of Ophthalmology 157, 1064-1069.

677 Zinkernagel, M.S., Schorno, P., Ebneter, A., Wolf, S., 2015. Scleral thinning after

678 repeated intravitreal injections of antivascular endothelial growth factor agents in the

679 same quadrant. Investigative Ophthalmology and Visual Science 56, 1894-1900.

680 
681

682

683

684

\section{Figure Captions}

Figure 1. Aerial view of the Badal optometer which was mounted in front of the anterior segment objective lens of the Spectralis AS-OCT. The temporal sclera of the participant's left eye was measured while they viewed a Maltese cross displayed on an LCD screen imaged through a +13 D Badal optometer which allowed for the correction of each participant's ametropia, and to provide accommodation stimuli at 0,3 and $6 \mathrm{D}$. The right eye was occluded for the duration of the task.

Figure 2. A) En-face image showing the scanning protocol for obtaining anterior scleral images with the AS-OCT. A $20^{\circ} \times 5^{\circ}$ volume scan with 21 lines of 30 averaged B-scans was used to obtain images of the anterior temporal sclera utilising the instrument's EDI technique. B) A typical B-scan of the anterior temporal sclera with anatomical landmarks labelled. C) B-scan with automated segmentation, showing the anterior conjunctiva marked in red and the posterior sclera in green. The blue line represents the anterior scleral boundary, which required manual segmentation. The vertical red line marks the position of the scleral spur. Scleral thickness was measured between the anterior scleral and posterior scleral boundary, and total wall thickness was measured between the anterior conjunctival boundary and posterior scleral boundary.

Figure 3. Overview of the OCT image analysis procedures used to determine scleral thickness and total wall thickness. For simplicity, the $6 \mathrm{D}$ scan analysis procedure has been omitted. A) Three common anatomical landmarks were chosen on the $0 \mathrm{D}$ (baseline) enface image and subsequent $3 \mathrm{D}$ and $6 \mathrm{D}$ scans in order to rotationally and translationally align the scans with the baseline image. B) The scan line corresponding to the position of the central line scan of the baseline $(0 \mathrm{D})$ en-face image was identified in the $3 \mathrm{D}$ scan (red line). C) The 5 central line scans in the $0 \mathrm{D}$ scan (and corresponding line scans in the $3 \mathrm{D}$ scan) were then analysed to segment the anterior conjunctival boundary (red), anterior scleral boundary (blue), and posterior scleral boundary (green). A vertical reference line (shown by the solid white line) was placed to mark the position of the scleral spur (SS) in each image. D) Data obtained via the segmentation of the 5 central line scans was then used to derive scleral thickness volume maps for each subject at each accommodation demand. These thickness volume maps were then rotated to align precisely with the baseline $(0 D)$ map, and a thickness profile from the corresponding location of the $0 D$ central line, in each of the final rotated thickness volumes was extracted and analysed. 
715 Scleral thickness measurements were then taken at discrete points 1,2 and $3 \mathrm{~mm}$ posterior

716 to the scleral spur (white dotted lines).

717

718 Figure 4. Change in average scleral thickness and total wall thickness (mean \pm SEM, $\mu \mathrm{m}$ )

719 from baseline for all subjects to the $3 \mathrm{D}$ and $6 \mathrm{D}$ stimulus. Data points marked with a cross

$720(\dagger)$ indicate a significant change from baseline $(p<0.05)$. Negative values indicate a

721 thinning with accommodation.

722

723 Figure 5. Change in scleral thickness ( $A$ and $B)$ (mean $\pm S E M, \mu m)$ and change in total wall 724 thickness ( $C$ and $D$ ) (mean \pm SEM, $\mu \mathrm{m}$ )from baseline at points 1,2 and $3 \mathrm{~mm}$ posterior to 725 the scleral spur in the myopic $(n=18)$ and emmetropic $(n=15)$ subjects, to the $3 \mathrm{D}$ ( $\mathrm{A}$ and

$726 \mathrm{C})$ and $6 \mathrm{D}(\mathrm{B}$ and $\mathrm{D})$ stimulus. Data points marked with a cross $(\mathrm{t})$ indicate a significant 727 change from baseline $(p<0.05)$, and those marked with an asterisk $\left(^{*}\right)$ indicate a highly 728 significant change from baseline $(p<0.001)$. Negative values indicate a thinning with 729 accommodation, and positive values indicate a thickening. 


\begin{tabular}{|c|c|c|c|c|c|c|}
\hline & \multicolumn{3}{|c|}{$\begin{array}{l}\text { Scleral Thickness } \\
\text { (mean } \pm \text { SD, } \mu \mathrm{m} \text { ) }\end{array}$} & \multicolumn{3}{|c|}{$\begin{array}{l}\text { Total Wall Thickness } \\
\qquad(\text { mean } \pm S D, \mu \mathrm{m})\end{array}$} \\
\hline & $1 \mathrm{~mm}$ & $2 \mathrm{~mm}$ & $3 \mathrm{~mm}$ & $1 \mathrm{~mm}$ & $2 \mathrm{~mm}$ & $3 \mathrm{~mm}$ \\
\hline All subjects & $512 \pm 52$ & $504 \pm 60$ & $543 \pm 67$ & $751 \pm 81$ & $730 \pm 87$ & $752 \pm 91$ \\
\hline Myopes & $513 \pm 62$ & $505 \pm 72$ & $544 \pm 73$ & $751 \pm 86$ & $726 \pm 89$ & $743 \pm 91$ \\
\hline Emmetropes & $511 \pm 39$ & $503 \pm 43$ & $542 \pm 62$ & $751 \pm 79$ & $735 \pm 87$ & $763 \pm 92$ \\
\hline
\end{tabular}

732 Table 1. Baseline scleral thickness (mean $\pm \mathrm{SD}, \mu \mathrm{m}$ ) and total wall thickness (mean $\pm \mathrm{SD}$,

$733 \mu \mathrm{m})$ at points 1,2 and $3 \mathrm{~mm}$ posterior to the scleral spur in all subjects $(n=33)$, myopes $(n=$ 734 18) and emmetropes $(n=15)$. 


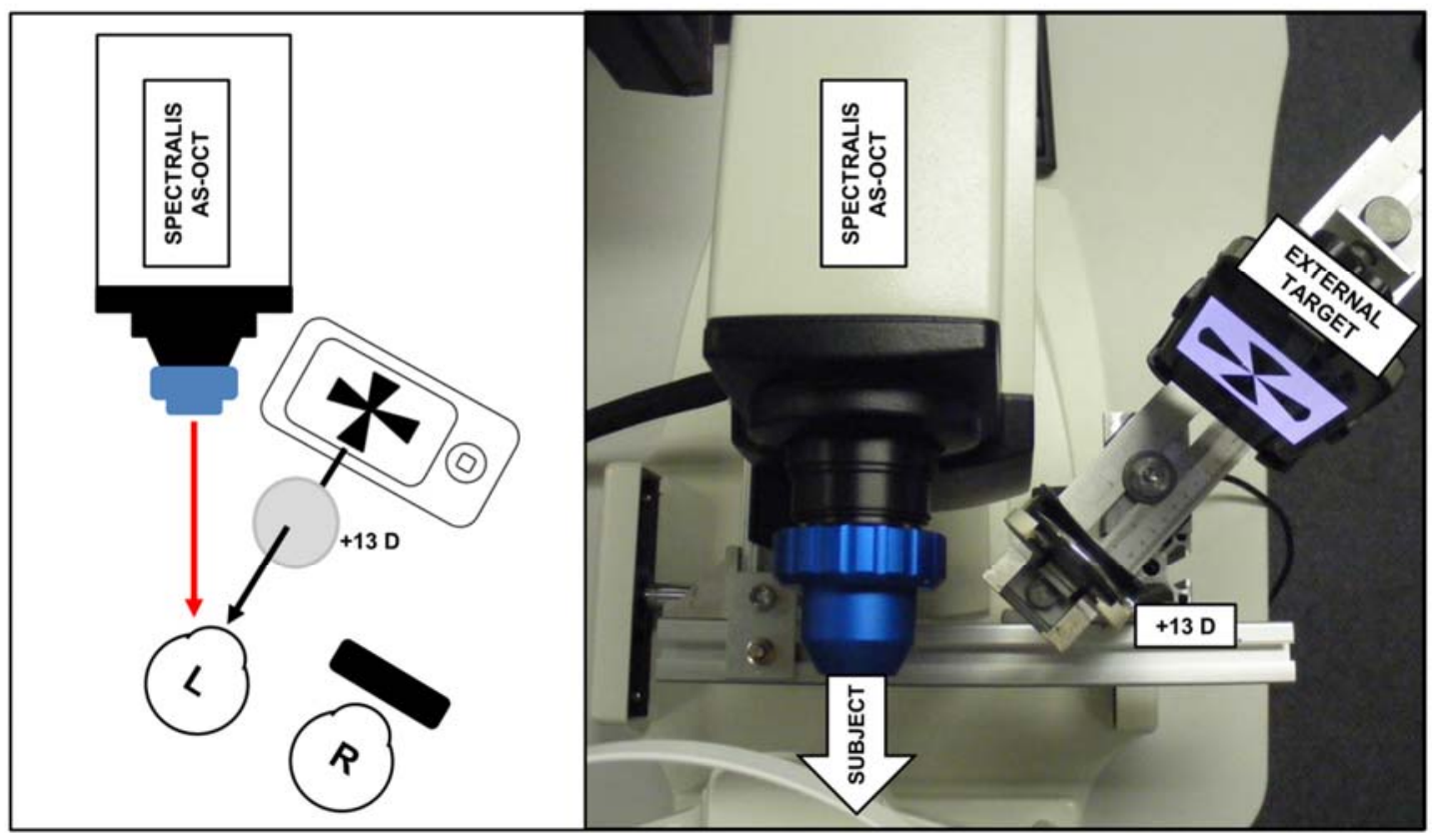

735

736 Figure 1. Aerial view of the Badal optometer which was mounted in front of the anterior

737 segment objective lens of the Spectralis AS-OCT. The temporal sclera of the participant's

738 left eye was measured while they viewed a Maltese cross displayed on an LCD screen

739 imaged through a +13 D Badal optometer which allowed for the correction of each

740 participant's ametropia, and to provide accommodation stimuli at 0,3 and $6 \mathrm{D}$. The right eye

741 was occluded for the duration of the task. 


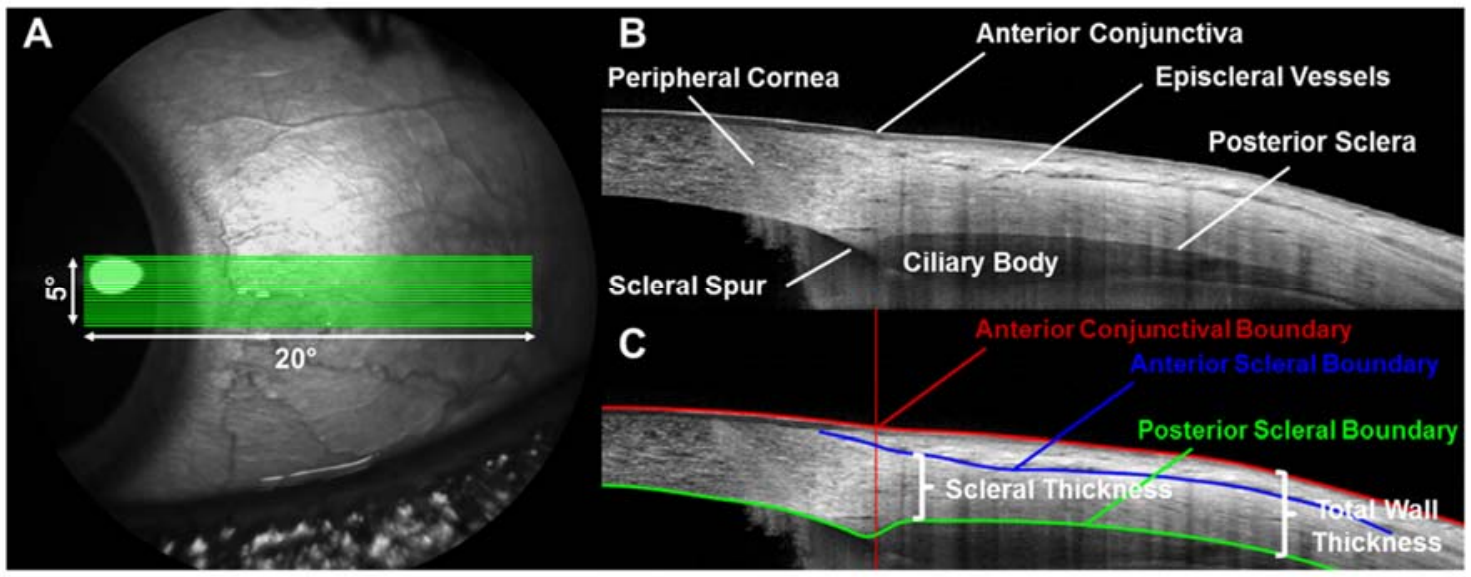

743 Figure 2. A) En-face image showing the scanning protocol for obtaining anterior scleral

744 images with the AS-OCT. A $20^{\circ} \times 5^{\circ}$ volume scan with 21 lines of 30 averaged B-scans was

745 used to obtain images of the anterior temporal sclera utilising the instrument's EDI

746 technique. B) A typical B-scan of the anterior temporal sclera with anatomical landmarks

747 labelled. C) B-scan with automated segmentation, showing the anterior conjunctiva marked

748 in red and the posterior sclera in green. The blue line represents the anterior scleral

749 boundary, which required manual segmentation. The vertical red line marks the position of

750 the scleral spur. Scleral thickness was measured between the anterior scleral and posterior

751 scleral boundary, and total wall thickness was measured between the anterior conjunctival

752 boundary and posterior scleral boundary. 


\section{O D SCAN}

\section{D SCAN}

A En-face Image Analysis
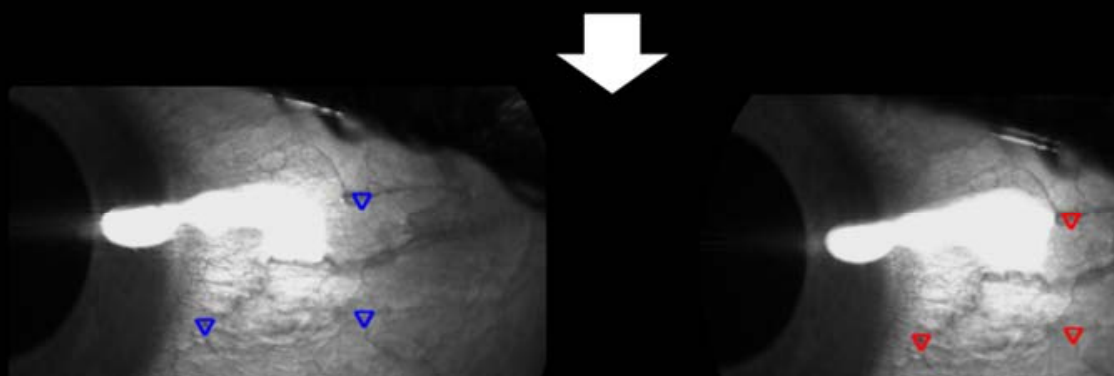

B Identification of Corresponding Line Scans

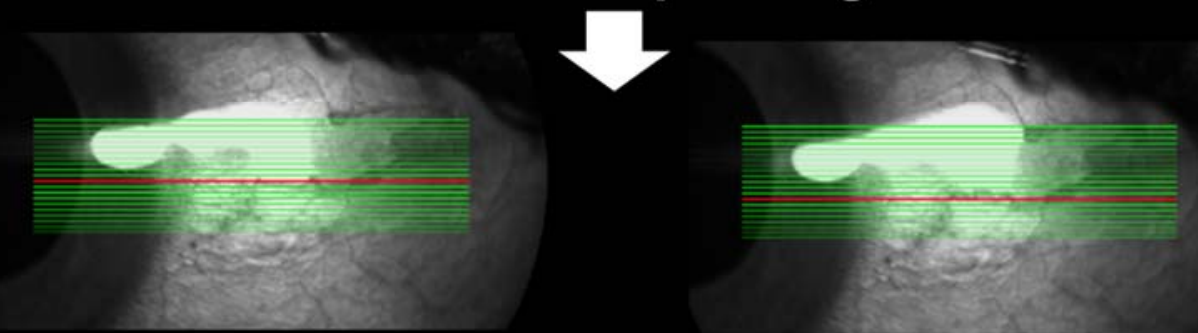

C Segmentation of 5 Central Line Scans

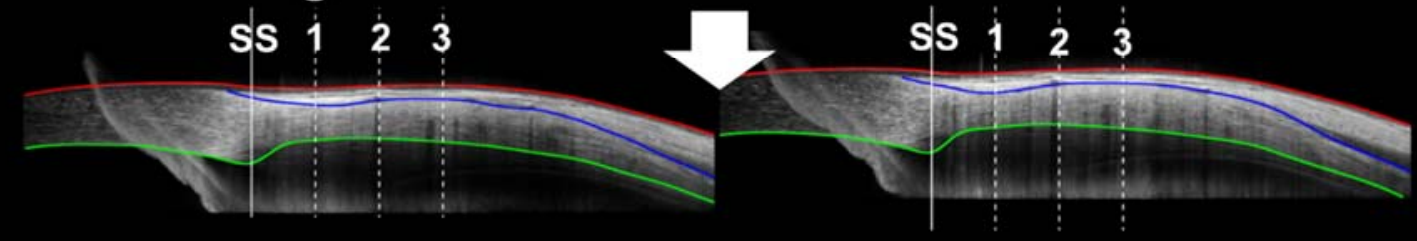

D Generation of Scleral Thickness Profiles

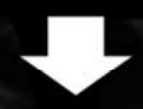

Figure 3. Overview of the OCT image analysis procedures used to determine scleral thickness and total wall thickness. For simplicity, the $6 \mathrm{D}$ scan analysis procedure has been omitted. A) Three common anatomical landmarks were chosen on the $0 \mathrm{D}$ (baseline) enface image and subsequent $3 \mathrm{D}$ and $6 \mathrm{D}$ scans in order to rotationally and translationally align the scans with the baseline image.

B) The scan line corresponding to the position of 
759 the central line scan of the baseline (OD) en-face image was identified in the $3 \mathrm{D}$ scan (red

760 line). C) The 5 central line scans in the $0 \mathrm{D}$ scan (and corresponding line scans in the $3 \mathrm{D}$

761 scan) were then analysed to segment the anterior conjunctival boundary (red), anterior

762 scleral boundary (blue), and posterior scleral boundary (green). A vertical reference line

763 (shown by the solid white line) was placed to mark the position of the scleral spur (SS) in

764 each image. D) Data obtained via the segmentation of the 5 central line scans was then

765 used to derive scleral thickness volume maps for each subject at each accommodation

766 demand. These thickness volume maps were then rotated to align precisely with the

767 baseline (OD) map, and a thickness profile from the corresponding location of the $0 \mathrm{D}$

768 central line, in each of the final rotated thickness volumes was extracted and analysed.

769 Scleral thickness measurements were then taken at discrete points 1,2 and $3 \mathrm{~mm}$ posterior

770 to the scleral spur (white dotted lines). 


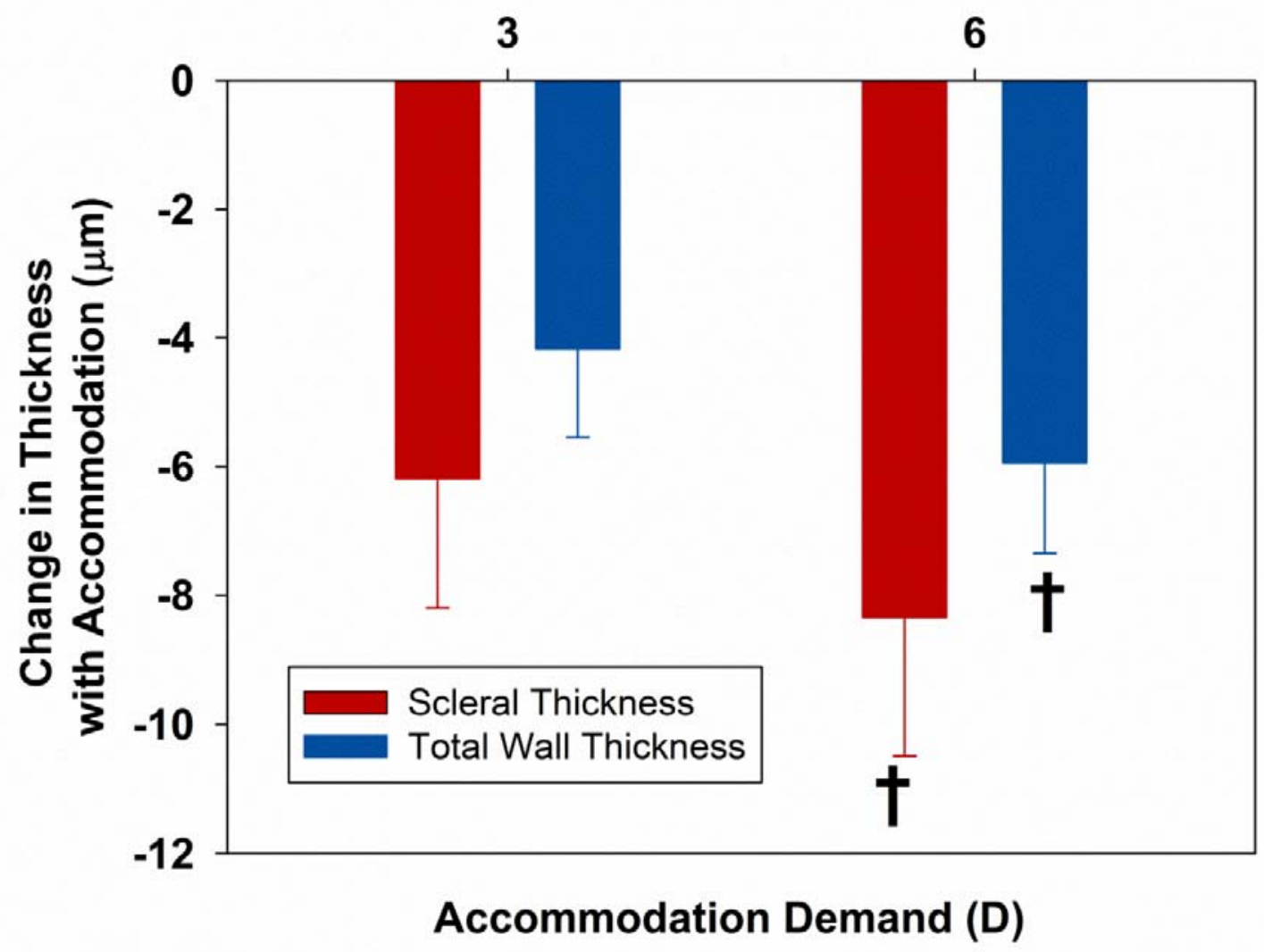

772 Figure 4. Change in average scleral thickness and total wall thickness (mean $\pm \mathrm{SEM}, \mu \mathrm{m}$ )

773 from baseline for all subjects to the $3 \mathrm{D}$ and $6 \mathrm{D}$ stimulus. Data points marked with a cross

$774(\dagger)$ indicate a significant change from baseline $(p<0.05)$. Negative values indicate a

775 thinning with accommodation. 


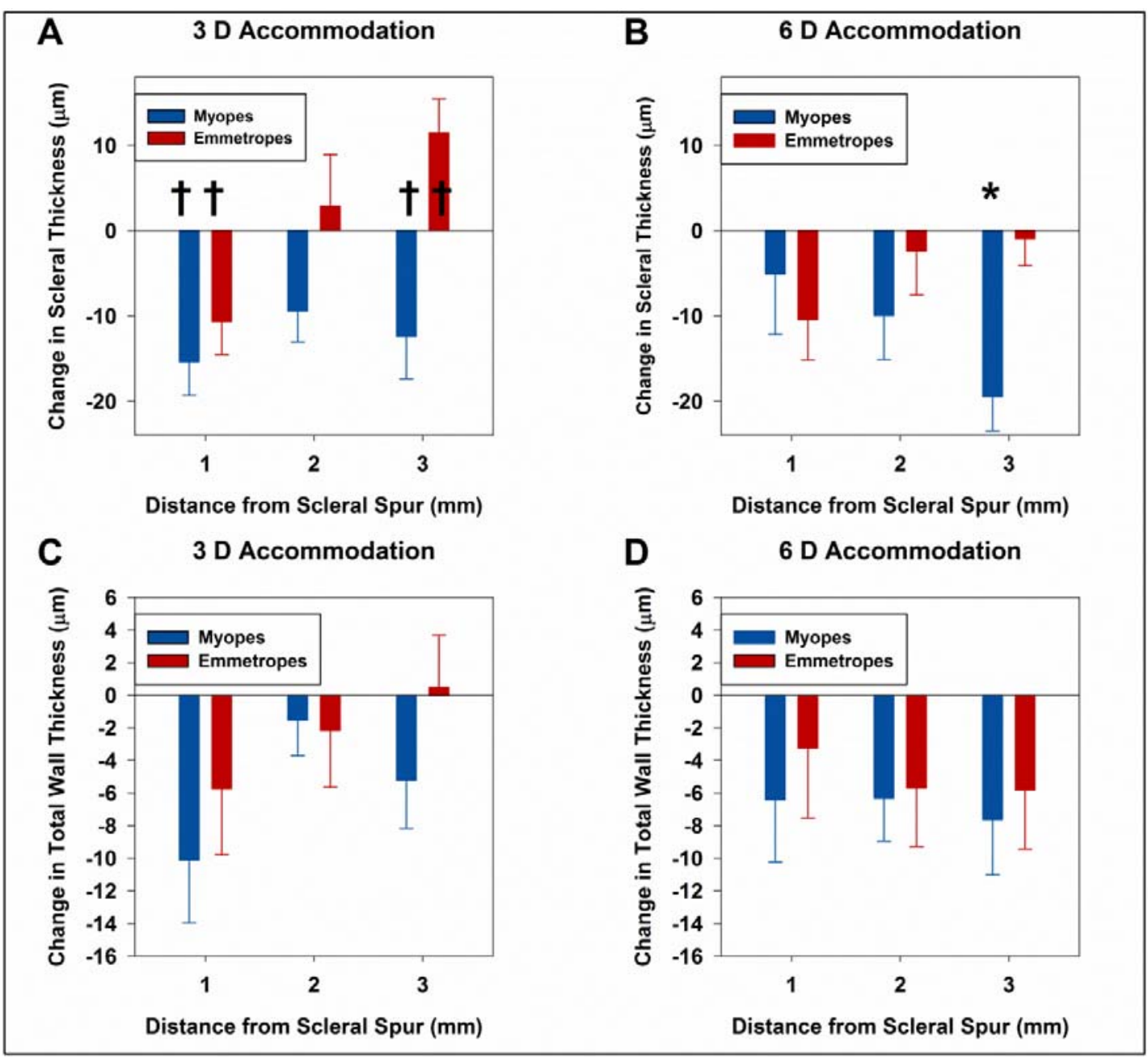

778 Figure 5. Change in scleral thickness ( $A$ and $B)$ (mean \pm SEM, $\mu \mathrm{m})$ and change in total wall 779 thickness $(C$ and $D)$ (mean \pm SEM, $\mu \mathrm{m}$ ) from baseline at points 1, 2 and $3 \mathrm{~mm}$ posterior to 780 the scleral spur in the myopic $(n=18)$ and emmetropic $(n=15)$ subjects, to the $3 \mathrm{D}(\mathrm{A}$ and 781 C) and $6 \mathrm{D}(\mathrm{B}$ and $\mathrm{D})$ stimulus. Data points marked with a cross $(\boldsymbol{\dagger})$ indicate a significant 782 change from baseline $(p<0.05)$, and those marked with an asterisk $\left({ }^{*}\right)$ indicate a highly 783 significant change from baseline $(p<0.001)$. Negative values indicate a thinning with 784 accommodation, and positive values indicate a thickening. 